\title{
Wilms tumor in patients with osteopathia striata with cranial sclerosis
}

\author{
Alicia Bach $\left(\mathbb{D}^{1}\right.$ - Jingyi $\mathrm{Mi}^{2} \cdot$ Matthew Hunter ${ }^{3,4}$ - Benjamin J. Halliday ${ }^{2} \cdot$ Sixto García-Miñaúr ${ }^{5}$. \\ Francesca Sperotto $\mathbb{1}^{6} \cdot$ Eva Trevisson $\mathbb{1}^{7,8} \cdot$ David Markie $\mathbb{D}^{9} \cdot \operatorname{lan}$ M. Morison ${ }^{9} \cdot$ Marwan Shinawi $\mathbb{B}^{10}$. \\ Daniel N. Willis $\mathbb{1}^{1} \cdot$ Stephen P. Robertson $\mathbb{1}^{2}$
}

Received: 2 June 2020 / Revised: 12 August 2020 / Accepted: 21 August 2020 / Published online: 2 September 2020

(c) The Author(s), under exclusive licence to European Society of Human Genetics 2020

\begin{abstract}
Germline pathogenic variants in AMERI cause osteopathia striata with cranial sclerosis (OSCS: OMIM 300373), an X-linked sclerosing bone disorder. Female heterozygotes exhibit metaphyseal striations in long bones, macrocephaly, cleft palate, and, occasionally, learning disability. Male hemizygotes typically manifest the condition as fetal or neonatal death. Somatically acquired variants in AMERI are found in neoplastic tissue in 15-30\% of patients with Wilms tumor; however, to date, only one individual with OSCS has been reported with a Wilms tumor. Here we present four cases of Wilms tumor in unrelated individuals with OSCS, including the single previously published case. We also report the first case of bilateral Wilms tumor in a patient with OSCS. Tumor tissue analysis showed no clear pattern of histological subtypes. In Beckwith-Wiedemann syndrome, which has a known predisposition to Wilms tumor development, clinical protocols have been developed for tumor surveillance. In the absence of further evidence, we propose a similar protocol for patients with OSCS to be instituted as an initial precautionary approach to tumor surveillance. Further evidence is needed to refine this protocol and to evaluate the possibility of development of other neoplasms later in life, in patients with OSCS.
\end{abstract}

\section{Introduction}

An increasing number of Mendelian syndromes that present in infancy have tumor predisposition as a part of their clinical manifestations [1,2]. In children with Beckwith-Wiedemann syndrome (BWS), the estimated

These authors contributed equally: Alicia Bach, Jingyi Mi

Stephen P. Robertson

stephen.robertson@otago.ac.nz

1 Division of Hematology/Oncology, Department of Pediatrics, Washington University School of Medicine, St Louis, MI, USA

2 Department of Women's and Children's Health, Dunedin School of Medicine, University of Otago, Dunedin 9016, New Zealand

3 Monash Genetics, Monash Medical Centre, Melbourne, VIC, Australia

4 Department of Paediatrics, Monash University, Melbourne, VIC, Australia

5 Instituto de Genética Médica y Molecular, La Paz University Hospital, Madrid, Spain risk of tumor development is $7.5 \%$ [3], with most tumors occurring within the first 8-10 years of life [4]. The supposition that there is a clinical benefit in tumor screening in individuals with these syndromes assumes that the benefits of screening, such as improving patient survival through early detection and management, outweighs the financial cost and added stress for the patient and family. In BWS, although different molecular subtypes are associated with different tumor types and risks, a surveillance program for all categories of the condition (except patients

6 Department of Women's and Children's Health, Padova University, Padova, Italy

7 Institute of Pediatric Research Città della Speranza, Clinical Genetics and Epidemiology Laboratory, Padova University, Padova, Italy

8 Clinical Genetics Unit, Department of Women's and Children's Health, University of Padova, Padova, Italy

9 Department of Pathology, Dunedin School of Medicine, University of Otago, Dunedin 9016, New Zealand

10 Division of Genetics and Genomic Medicine, Department of Pediatrics, Washington University School of Medicine, St Louis, MI, USA 
with isolated IC2 loss of methylation) has been recommended for consistency [5].

Osteopathia striata with cranial sclerosis (OSCS: OMIM 300373) is an X-linked condition that manifests in females who are heterozygous for pathogenic variants in AMERI (OMIM 300647). Females typically present with osteosclerosis with metaphyseal striations, macrocephaly, cleft palate, and, occasionally, learning disability. In males, the condition is often fatal due to multiple malformations affecting the cardiovascular and gastrointestinal systems. Somatic pathogenic variants in AMERI are found in neoplastic tissue in $15-30 \%$ of patients with Wilms tumor $[6,7]$, a surprising finding given that cohorts of patients with OSCS, including adults, have demonstrated no association between OSCS and Wilms tumor [8,9]. This paradox may be explained by germline pathogenic variants in AMERI promoting proliferation of nephrogenic rests, which have a low but definite rate of progression to Wilms tumor [10]. In this report, we present four unrelated female patients with OSCS who have been diagnosed with Wilms tumor, including the previously published case [11]. This association between OSCS and Wilms tumor, although anecdotal, could justify the initiation of screening for Wilms tumor in individuals diagnosed with this syndrome.

\section{Subjects and methods}

Three unrelated individuals with OSCS, who developed Wilms tumor, were ascertained by physician-initiated referral (Table 1) and are described here alongside the sole previously reported individual [11].

\section{Ethics statement}

Informed consent was obtained from all patients including archived permission for use of the photograph in Fig. 1a. Written permission was not necessary for the other three de-identified patients. Case 2 is now deceased. Analysis was performed in accordance with relevant guidelines and regulations under approval from the Health and Disability Ethics Committee of NZ (13STH56).

\section{Case reports}

\section{Case 1}

This female, now aged 29, was born at 41 weeks' gestation. During infancy, macrocephaly (head circumference $52 \mathrm{~cm}$, $+3.0 \mathrm{SD}$ ) and dysmorphism (hypertelorism, notched lips, small ears, hypoplastic alae nasi, epicanthic folds) were noted in addition to micrognathia and cleft palate. Her skeletal survey showed striations in the long bones, proximal fibular hypoplasia, and a sclerotic skull base and vault. She developed hydrocephalus requiring insertion of a ventricular shunt, short stature (stature tracked consistently at $-2 \mathrm{SD}$ ), and scoliosis. She also had pectus excavatum, a ventricular septal defect, tracheomalacia, conductive hearing impairment, delayed exfoliation and eruption of primary and secondary dentition respectively, and mild developmental delay. As an adult, she has does not manifest intellectual disability.

At age 2 years, bilateral abdominal masses were detected with concurrent macroscopic hematuria. A computerized tomography (CT) scan and abdominal ultrasound scan confirmed bilateral well-defined renal masses involving the upper third of both kidneys with no evidence of perirenal invasion or retroperitoneal lymphadenopathy. She underwent subsequent bilateral resection, followed by chemotherapy.

Tumor histology demonstrated that the renal tissue was largely replaced by a mass of fibrous tissue, containing atypical but mature tubules and glomeruli, and necrotic tissue with occasional areas of calcification. Sections of renal parenchyma were histologically deranged with epithelial elements embedded in a dense, partly collagenized stroma. The epithelial elements consisted predominantly of dysplastic tubules but moderate numbers of glomeruloid bodies were also present. No blastemal tissue was detected. These findings were consistent with nephroblastomata with partial maturation.

At age 24 years, targeted molecular testing of AMERI revealed heterozygosity for a de novo whole gene deletion of AMER1, NC_000023.10: g.(63395050_63495049)del, with deletion of the flanking gene $A S B 12$ but preservation of ARHGEF9.

\section{Case 2}

This female was born during the 36th week of gestation. She required neonatal intensive care due to respiratory failure and feeding difficulties. She underwent tracheostomy for laryngomalacia and gastrostomy, and fundoplication for severe gastroesophageal reflux and dysphagia. She had a submucosal cleft palate, macrocephaly (head circumference +5 SD), hypertelorism, frontal bossing, and a small anteverted nose. She developed mild bilateral conductive hearing loss and exhibited global developmental delay with decreased cognitive, motor, and social skills. However, at the time of her diagnosis with Wilms tumor, there was no clinical concern about cognitive, social, or motor delays. Radiographs demonstrated pronounced calvarial osteosclerosis with thickening of diploic space of the frontal and occipital bones, multiple wormian bones, and metaphyseal striations. Targeted sequencing of the coding exon of AMERI revealed heterozygosity for a de novo nonsense variant, NM_152424.3: 
Table 1 Clinical characteristics of individuals with OSCS and Wilms tumor.

\begin{tabular}{|c|c|c|c|c|}
\hline Case & 1 & 2 & 3 & 4 \\
\hline \multicolumn{5}{|l|}{ Patient characteristics } \\
\hline Age at last follow-up & $29 \mathrm{y}$ & $12 \mathrm{y}$ & $1 \mathrm{y}$ & $7 \mathrm{y}$ \\
\hline Sex & $\mathrm{F}$ & $\mathrm{F}$ & $\mathrm{F}$ & $\mathrm{F}$ \\
\hline Age at diagnosis of WT & $2 \mathrm{y}$ & $12 \mathrm{y}$ & $7 \mathrm{~m}$ & $3 \mathrm{y}$ \\
\hline Age at diagnosis of OSCS & $24 \mathrm{y}$ & $3 y$ & $7 \mathrm{~m}$ & $3 y$ \\
\hline gDNA (NC_000023.10) & $\begin{array}{l}\text { g.(63395050_63495049) } \\
\text { del }\end{array}$ & g. $63412110 \mathrm{G}>\mathrm{A}$ & $\begin{array}{l}\text { g.(62855819_63499639) } \\
\text { del }\end{array}$ & g.63412611_63412612delCA \\
\hline cDNA (NM_152424.3) & No mRNA & c. $1057 \mathrm{C}>\mathrm{T}$ & No mRNA & c.555_556delTG \\
\hline Protein (NP_689637) & No protein & p. $\left(\operatorname{Arg} 353^{*}\right)$ & No protein & p.(Ala187*) \\
\hline De novo/familial & De novo & De novo & De novo & De novo \\
\hline \multicolumn{5}{|l|}{ Clinical features } \\
\hline $\begin{array}{l}\text { Birthweight }(\mathrm{g}) / \\
\text { gestation }(\mathrm{w})\end{array}$ & $3012 / 41$ & NA/36 & $2655 / 36$ & $3770 / 39$ \\
\hline Bony sclerosis & + & + & + & + \\
\hline Bony striations & + & + & + & + \\
\hline Hearing impairment & + & + & + & + \\
\hline Orofacial clefting & + & + & + & + \\
\hline Developmental delay & + & + & + & + \\
\hline Macrocephaly & + & + & + & + \\
\hline \multicolumn{5}{|l|}{ Tumor features } \\
\hline Anaplasia & - & Diffuse & - & - \\
\hline Histological composition & $\mathrm{PE}$ & $\mathrm{PB}$ & $\mathrm{PE}$ & $\mathrm{PB}$ \\
\hline Nephrogenic rests & - & - & - & - \\
\hline Relapse & - & - & - & - \\
\hline Stage at diagnosis & I (for each tumor) & III & I & III \\
\hline Laterality & Bilateral & Unilateral & Unilateral & Unilateral \\
\hline Metastases & - & 6/6 Retrocaval nodes & - & - \\
\hline
\end{tabular}

$m$ months, $N A$ not available, $P B$ predominantly blastemal, $P E$ predominantly epithelial, $w$ weeks, $y$ years.

Fig. 1 Clinical, radiographical and histological data from cases 2 and 3. a Photograph of case 3 showing typical features of OSCS including

macrocephaly, frontal bossing, hypertelorism, and a long philtrum. b Macrocephaly and cranial sclerosis on skull Xray in case 3. c Metaphyseal striations in the long bones of the lower limbs on X-ray from case 3. d Abdominal

computerized tomography scan obtained at diagnosis from case 2 showing a large right-sided renal mass, perinephric hematoma, and retrocaval lymphadenopathy. e H\&Estained histology from case 2 of resected tumor showing Wilms tumor with diffuse anaplasia.
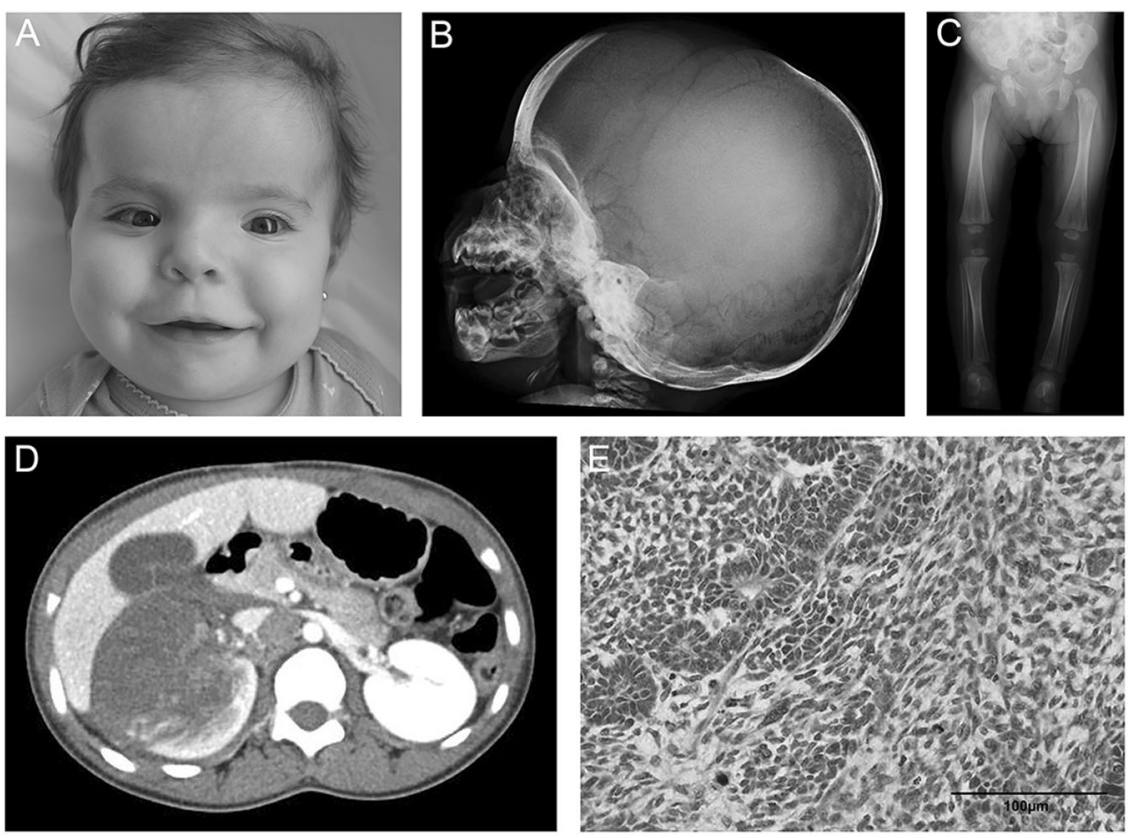
c.1057C > T (NP_689637: p.(Arg353*)), previously identified in other individuals with OSCS [8], and also as a somatically acquired event in Wilms tumor [7].

At age 12 years, she presented with 2-3 days of severe right-sided abdominal pain and fever. Abdominal CT revealed a renal mass with significant perinephric hematoma and retrocaval lymphadenopathy (Fig. 1d). Chest CT was negative for metastases. CT-guided biopsy confirmed the mass to be a predominantly blastemal stage III Wilms tumor. Primary nephrectomy was deferred due to tumor rupture and hemorrhage. A right radical nephrectomy with lymph node dissection was performed after 5 weeks of chemotherapy and minimal response to treatment. Pathology revealed diffuse anaplasia with no associated nephrogenic rests (Fig. 1e). Retrocaval nodes (6/6) were positive for tumor. While still receiving chemotherapy, the patient developed numerous pulmonary metastases and enlarging abdominal lymphadenopathy. She then underwent treatment with phase I investigational agents but subsequently died from tumor progression.

\section{Case 3}

The female was born during the 36th week of gestation. She presented with macrocephaly (head circumference $47 \mathrm{~cm}$, $+1.29 \mathrm{SD}$ at 9 months), hypotonia, cleft soft palate, facial dysmorphism, and developmental delay (Fig. 1a-c). Chromosomal microarray showed a de novo $644 \mathrm{~kb}$ whole gene deletion, NC_000023.10: g.(62855819_63499639)del, encompassing AMERI and ARHGEF9 at the proximal end of the deletion, and MTMR 8 distally. At 7 months of age, she was diagnosed with a stage I Wilms tumor after abdominal ultrasound scan detected a left-sided renal mass as part of clinical investigations following the microarray result. The tumor was non-anaplastic and predominantly epithelial in its histology without associated nephrogenic rests. She underwent tumor nephrectomy at 8 months, followed by chemotherapy. Skeletal survey showed characteristic radiological findings, including metaphyseal striations in the long bones of the lower limb and cranial sclerosis on skull X-ray.

\section{Case 4}

This female patient has been previously described by Sperotto et al. [11]. The patient was born in the 39th week of gestation and exhibited features including macrocephaly (head circumference $38.5 \mathrm{~cm},+2.0 \mathrm{SD}$ ), cleft palate, frontal bossing, hypertelorism, retrognathia, anteverted nares, and bilateral second-toe clinodactyly. She developed a mild cognitive impairment and hearing loss, and received bilateral cochlear implants at the age of 2 years. The patient was formally diagnosed with OSCS after direct sequencing of
AMERl revealed a heterozygous de novo nonsense variant designated as NM_152424.3: c.555_556delTG (NP_68963 7.3: (p.Ala187*)).

At 36 months of age, the patient presented with a painless abdominal mass and was diagnosed with a stage III Wilms tumor. The tumor was non-anaplastic and predominantly blastemal. She was started on neoadjuvant chemotherapy, after which the tumor became the regressive type, with $90 \%$ necrosis and $10 \%$ residual blastemal cells. Due to minimal liver infiltration revealed by histological examination on follow-up, she underwent nephrectomy, adjuvant chemotherapy, and focal liver radiotherapy. The patient, now 7 years of age, has remained in remission. At clinical assessment at 6 years of age, she had severe intellectual disability with absent speech. At the most recent clinical follow-up at 7 years, she manifested adequate growth but had persisting expressive language delay. She had had cochlear implants inserted for moderate conductive deafness during toddlerhood and had delayed eruption of the permanent teeth, with persistence of deciduous dentition.

\section{Discussion}

We report four cases of Wilms tumor in individuals with OSCS and propose a likely association between these two rare conditions. Germline loss-of-function or truncating pathogenic variants in AMERl cause OSCS [8], and somatic variants in $A M E R 1$ are found in $\sim 15-30 \%$ of sporadic Wilms tumors [6, 7]. AMERl regulates WNT signaling and induces ubiquitination and degradation of $\beta$-catenin $[12,13]$. As aberrant activation of WNT signaling promotes tumorigenesis [14, 15], AMERl has been designated a tumor suppressor gene [13]. The prevalence of OSCS is estimated to be $<1$ in 1 million with fewer than 100 cases reported in literature [16]. The incidence of Wilms tumor is 1 in 10,000 infants [17].

From our study, there appears to be an overrepresentation of Wilms tumor in OSCS patients. Jenkins et al. [8] proposed that the process of tumor development may require a specific temporal or spatial patterning in the acquisition of the genetic and epigenetic factors (such as pathogenic variants in CTNNB1 and WT1) to result in tumorigenesis, and that an individual with an AMERI pathogenic variant alone may not be predisposed to tumorigenesis for this reason [8, 9, 18]. However, more recently, several instances of different tumor types have been described in individuals with OSCS including childhood hepatoblastoma [19], and ovarian [9] and colorectal carcinoma [8] in adulthood.

In this cohort study, there was no clear pattern of age of onset of the tumors, with age of diagnosis ranging from 
7 months to 12 years. Many tumor predisposition syndromes caused by pathogenic variants in tumor suppressor genes demonstrate a higher preponderance of bilateral disease. For sporadic presentations, 5-7\% of Wilms tumors present bilaterally, either synchronously or metachronously [20], but only one individual in our OSCS cohort presented with bilateral disease. Similarly, there was no clear pattern for the histological subtype for tumors that arose in association with OSCS (Table 1). Somatic AMERI variants are found in $15-30 \%$ of Wilms tumors, evenly split between tumors with either mesenchymal or epithelial nephrogenic histology [21]. At this early stage in our understanding of the association between Wilms tumor and OSCS, we cautiously conclude that germline pathogenic variants in AMERI do not seem to be associated with any one histological subtype of tumor.

Fukuzawa et al. [10] proposed that pathogenic variants in AMERI may predispose to expansion of nephrogenic rests rather than directly promote the development of Wilms tumor itself. As only $1 \%$ of rests progress onto tumor development [10] and $0.9 \%$ of otherwise healthy children have histologically identifiable nephrogenic rests in early infancy [22], these figures may explain the relative rarity of Wilms tumor even in individuals with a diagnosis of OSCS.

In other conditions with predisposition to Wilms tumor, such as BWS, clinical protocols have been developed for tumor surveillance [18]. Although the genetic and epigenetic underpinnings of the tumor predisposition are likely to be fundamentally different to those in OSCS, we suggest that it may be prudent to consider tumor surveillance for children with a diagnosis of OSCS. The Pediatric Cancer Working Group of the American Association for Cancer Research considers a $\geq 5 \%$ chance of childhood tumor development as a reasonable threshold to recommend screening [23]. Surveillance for conditions with a cancer risk between $1-5 \%$ is supported if the screening modalities are relatively cost-effective and non-invasive, or if significantly poor patient outcomes for clinically detected tumors renders early detection worthwhile [23]. The survival rate for Wilms tumor has increased from $20 \%$ in the 1960 s to over $90 \%$ currently [24, 25]. Although the survival rate of children with BWS and Wilms tumor is not significantly different from those without BWS, children with BWS and Wilms tumor are more often diagnosed with early-stage disease; thus, screening allows for reduction in treatment-related morbidity [5]. In the absence of further evidence, a conservative approach would be to implement a surveillance protocol similar to that recommended for BWS $[23,26]$, namely abdominal ultrasound scan every 3 months until age 7 years [5]. The cut-off age for surveillance of children with BWS at 7 years is arbitrary; however, after the age of 10 years, tumor risk approaches the baseline cancer risk of the general population [27]. For instance, in the United States, two-thirds of cases of Wilms tumor are diagnosed before 5 years of age and $95 \%$ before 10 years [28]. Children with bilateral tumors are diagnosed earlier than those with unilateral disease [29]. However, as one out of the four patients in our case series were older than 10 years at diagnosis of Wilms tumor, patients with OSCS may have a prolonged risk for tumor development and may require continued screening beyond the age of 7 years. A firmer evidential basis for instituting this protocol for children with OSCS should continue to be sought, as should the possibility that these individuals may have a propensity to develop other neoplasms later on in life.

Acknowledgements We thank the individuals and their families described in this report. SPR is supported by Curekids. ET is supported by Istituto di Ricerca Pediatrica (IRP).

\section{Compliance with ethical standards}

Conflict of interest The authors declare that they have no conflict of interest.

Publisher's note Springer Nature remains neutral with regard to jurisdictional claims in published maps and institutional affiliations.

\section{References}

1. Gripp KW. Tumor predisposition in Costello syndrome. Am J Med Genet C Semin Med Genet. 2005;137C:72-7.

2. Weksberg R, Nishikawa J, Caluseriu O, Fei Y-L, Shuman C, Wei C, et al. Tumor development in the Beckwith-Wiedemann syndrome is associated with a variety of constitutional molecular $11 \mathrm{p} 15$ alterations including imprinting defects of KCNQ1OT1. Hum Mol Genet. 2001;10:2989-3000.

3. Pettenati MJ, Haines JL, Higgins RR, Wappner RS, Palmer CG, Weaver DD. Wiedemann-Beckwith syndrome: presentation of clinical and cytogenetic data on 22 new cases and review of the literature. Hum Genet. 1986;74:143-54.

4. DeBaun MR, Siegel MJ, Choyke PL. Nephromegaly in infancy and early childhood: a risk factor for Wilms tumor in BeckwithWiedemann syndrome. J Pediatrics. 1998;132:401-4.

5. Brioude F, Kalish JM, Mussa A, Foster AC, Bliek J, Ferrero GB, et al. Clinical and molecular diagnosis, screening and management of Beckwith-Wiedemann syndrome: an international consensus statement. Nat Rev Endocrinol. 2018;14:229-49.

6. Ruteshouser EC, Robinson SM, Huff V. Wilms tumor genetics: mutations in WT1, WTX, and CTNNB1 account for only about one-third of tumors. Genes Chromosomes Cancer. 2008; 47:461-70

7. Rivera MN, Kim WJ, Wells J, Driscoll DR, Brannigan BW, Han M. et al. An X chromosome gene, WTX, is commonly inactivated in Wilms tumor. Science. 2007;315:642-5.

8. Jenkins ZA, van Kogelenberg M, Morgan T, Jeffs A, Fukuzawa R, Pearl E, et al. Germline mutations in WTX cause a sclerosing skeletal dysplasia but do not predispose to tumorigenesis. Nat Genet. 2009;41:95-100.

9. Perdu B, Freitas FD, Frints SG, Schouten M, Schrander-Stumpel C, Barbosa M, et al. Osteopathia striata with cranial sclerosis owing to WTX gene defect. J Bone Miner Res. 2010;25:82-90. 
10. Fukuzawa R, Holman SK, Chow CW, Savarirayan R, Reeve AE, Robertson SP. WTX mutations can occur both early and late in the pathogenesis of Wilms tumour. J Med Genet. 2010;47:791-4.

11. Sperotto F, Bisogno G, Opocher E, Rossi S, Rigon C, Trevisson $\mathrm{E}$, et al. Osteopathia striata with cranial sclerosis and Wilms tumor: coincidence or consequence? Clin Genet. 2017;92:674-5.

12. Tanneberger K, Pfister AS, Kriz V, Bryja V, Schambony A, Behrens J. Structural and functional characterization of the Wnt inhibitor APC membrane recruitment 1 (Amer1). J Biol Chem. 2011;286:19204-14.

13. Major MB, Camp ND, Berndt JD, Yi X, Goldenberg SJ, Hubbert C. et al. Wilms tumor suppressor WTX negatively regulates $\mathrm{WNT} / \beta$ catenin signaling. Science. 2007;316:1043-6.

14. Lustig B, Behrens J. The Wnt signaling pathway and its role in tumor development. J Cancer Res Clin Oncol. 2003;129:199-221.

15. Clevers $H$. Wnt/ $\beta$-catenin signaling in development and disease. Cell. 2006;127:469-80.

16. Berenholz L, Lippy W, Harrell M. Conductive hearing loss in osteopathia striata-cranial sclerosis. Otolaryngol Head Neck Surg. 2002;127:124-6.

17. Howlader N, Noone AM, Krapcho M, Miller D, Brest A, Yu M, et al. (eds). SEER Cancer Statistics Review, 1975-2016 (Bethesda, MD: National Cancer Institute, 2019).

18. Weksberg R, Shuman C, Beckwith JB. Beckwith-Wiedemann syndrome. Eur J Hum Genet. 2010;18:8-14.

19. Fujita A, Ochi N, Fujimaki H, Muramatsu H, Takahashi Y, Natsume $\mathrm{J}$, et al. A novel WTX mutation in a female patient with osteopathia striata with cranial sclerosis and hepatoblastoma. Am J Med Genet A. 2014;164:998-1002.
20. Özyörük D, Emir S. The management of bilateral Wilms tumor. Transl Pediatr. 2014;3:34-8.

21. Fukuzawa R, Anaka MR, Weeks RJ, Morison IM, Reeve AE. Canonical WNT signalling determines lineage specificity in Wilms tumour. Oncogene. 2009;28:1063-75.

22. Beckwith JB, Kiviat NB, Bonadio JF. Nephrogenic rests, nephroblastomatosis, and the pathogenesis of Wilms' tumor. Pediatr Pathol. 1990;10:1-36.

23. Brodeur GM, Nichols KE, Plon SE, Schiffman JD, Malkin D. Pediatric cancer predisposition and surveillance: an overview, and a tribute to Alfred G. Knudson Jr. Clin Cancer Res. 2017;23:e1-e5.

24. Metzger ML, Dome JS. Current therapy for Wilms' tumor. Oncologist 2005;10:815-26.

25. Tournade MF, Com-Nougué C, de Kraker J, Ludwig R, Rey A, Burgers JMB, et al. Optimal duration of preoperative therapy in unilateral and nonmetastatic Wilms' tumor in children older than 6 months: results of the Ninth International Society of Pediatric Oncology Wilms' Tumor Trial and Study. J Clin Oncol. 2001; 19:488-500.

26. Craft AW. Growth rate of Wilms' tumour. Lancet 1999;354:1127.

27. DeBaun MR, Tucker MA. Risk of cancer during the first four years of life in children from The Beckwith-Wiedemann Syndrome Registry. J Pediatrics. 1998;132:398-400.

28. Ries LAG, Smith MA, Gurney JG, Linet M, Tamra T, Young JL, et al. (eds). Cancer Incidence and Survival among Children and Adolescents: United States SEER Program 1975-1995 (Bethesda, MD: National Cancer Institute, 1999).

29. Breslow N, Olshan A, Beckwith JB, Green DM. Epidemiology of Wilms tumor. Med Pediatr Oncol. 1993;21:172-81. 